\title{
Gasto catastrófico en salud en México y sus factores determinantes, 2002-2014
}

\author{
Román Rodríguez-Aguilar' y Gustavo Rivera-Peña ${ }^{2}$ \\ ${ }^{1}$ Facultad de Ingeniería; ${ }^{2}$ Facultad de Economía y Negocios, Universidad Anáhuac México. Estado de México, México
}

\section{Resumen}

Objetivo: Evaluar la protección financiera del aseguramiento público de salud mediante el análisis del porcentaje de hogares con gasto catastrófico en salud (HGCS) en México y su relación con la condición de pobreza, el tamaño de la localidad, la entidad federativa, la condición de aseguramiento y los rubros de gasto en salud. Método: Se utilizó la Encuesta Nacional de Ingreso y Gasto de los Hogares 2002-2014 para estimar el porcentaje de HGCS. Mediante un modelo probit se identificaron los factores asociados a la ocurrencia de gasto catastrófico. El análisis se realizó con Stata-SE 12. Resultados: En 2014 había un 2.08\% de HGCS (1.82-2.34\%; $N=657,474)$. El modelo probit estimado clasificó correctamente el $98.2 \%$ de los HGCS $(\operatorname{Pr}(D) \geq 0.5)$. Los factores que incidieron en el gasto catastrófico fueron la afiliación, la presencia de enfermedades crónicas, el gasto en hospitalización, la condición rural y que el hogar estuviera por debajo de la línea de pobreza alimentaria. Conclusiones: El porcentaje de HGCS disminuyó en los últimos años, mejorando la protección financiera en salud. Esta disminución pareciera haberse estancado, manteniendo inequidades en el acceso a servicios de salud, sobre todo en población rural, sin afiliación a alguna institución de salud, por debajo de la línea de pobreza alimentaria y con enfermedades crónicas.

PALABRAS CLAVE: Gasto en salud. Gasto de bolsillo. Modelos probabilísticos.

\begin{abstract}
Objective: To assess the financial protection of public health insurance by analyzing the percentage of households with catastrophic health expenditure (HCHE) in Mexico and its relationship with poverty status, size of locality, federal entity, insurance status and items of health spending. Method: Mexican National Survey of Income and Expenditures 2002-2014 was used to estimate the percentage of HCHE. Through a probit model, factors associated with the occurrence of catastrophic spending are identified. Analysis was performed using Stata-SE 12. Results: In 2014 there were 2.08\% of HCHE (1.82-2.34\%; $N=657,474)$. The estimated probit model correctly classified $98.2 \%$ of $H C H E(\operatorname{Pr}(D) \geq 0.5)$. Factors affecting the catastrophic expenditures were affiliation, presence of chronic disease, hospitalization expenditure, rural condition and that the household is below the food poverty line. Conclusions: The percentage of HCHE decreased in recent years, improving financial protection in health. This decline seems to have stalled, keeping inequities in access to health services, especially in rural population without affiliation to any health institution, below the food poverty line and suffering from chronic diseases.
\end{abstract}

KEY WORDS: Health expenditure. Out of pocket spending. Probabilistic models.

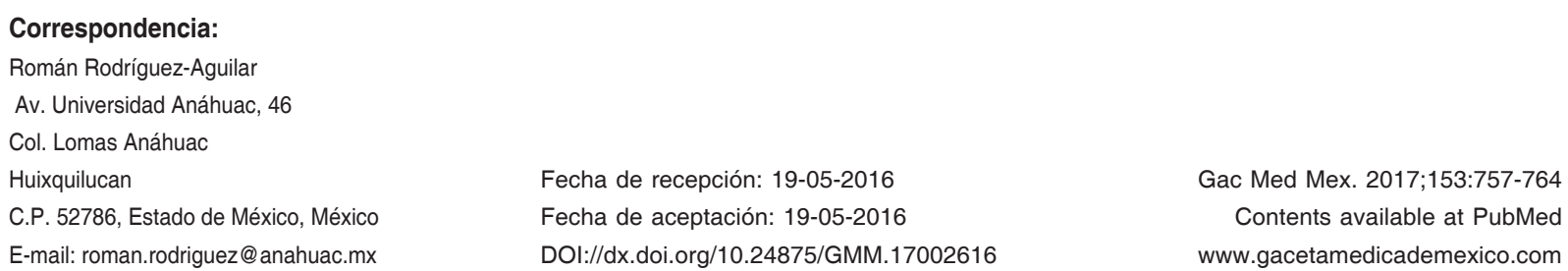

Fecha de recepción: 19-05-2016

Fecha de aceptación: 19-05-2016

E-mail: roman.rodriguez@anahuac.mx

DOI://dx.doi.org/10.24875/GMM.17002616

Gac Med Mex. 2017;153:757-764

Contents available at PubMed www.gacetamedicademexico.com 


\section{Introducción}

La fragmentación del Sistema de Salud en México ha propiciado que el financiamiento sea diferenciado y a través de diversas fuentes, para la población sin y con seguridad social. En el primer caso, el financiamiento proviene de impuestos generales, y en el segundo, de contribuciones obrero-patronales y gubernamentales a la seguridad social; permanece en ambos segmentos un componente de financiamiento a través del gasto de bolsillo (GB)'.

De acuerdo con los datos de la Organización para la Cooperación y el Desarrollo Económicos, el GB en México representa el $45 \%$ del gasto total en salud ${ }^{2}$, colocándonos en primer lugar entre los países miembros. Este mecanismo de financiamiento, por su naturaleza, puede conducir a un hogar a incurrir en gastos catastróficos (GC) por motivos de salud, generando altos costos sociales ${ }^{3}$. Se define a un hogar con GC en salud (HGCS) cuando destina el $30 \%$ o más de su ingreso disponible o capacidad de pago para atención de la salud. El ingreso disponible o la capacidad de pago ha sido definido por la Organización Mundial de la Salud (OMS) como el remanente del gasto total del hogar una vez descontadas sus necesidades básicas de subsistencia, medidas a través del gasto en alimentación ${ }^{4}$.

En México se han desarrollado diversos estudios para determinar la magnitud, el tipo y el número de HGCS. En 1992, la Fundación Mexicana para la Salud (FUNSALUD) señaló en un estudio que en México había un 2.3\% de HGCS. Otros estudios al respecto han determinado que, entre los años 1992 y 2000, el porcentaje de HGCS se situó entre el 3 y el $4 \%{ }^{5}$. Se ha documentado que los hogares que incurren en mayor medida en GC son aquellos que se encuentran en los quintiles de ingreso más bajo (alrededor del $10 \%)^{6}$. Ante este problema, a partir del año 2001, de manera gradual, se implementó el Sistema de Protección Social en Salud (SPSS), el cual tenía entre sus objetivos la disminución del GB y en especial del porcentaje de HGCS a través de una cobertura financiera otorgada a la población sin acceso a la seguridad social7. Los resultados observados después de la implementación del SPSS muestran que el GC ha disminuido; sin embargo, parece haberse estancado. En este contexto, el presente artículo pretende analizar la evolución del porcentaje de HGCS en México para el periodo 2002-2014. Adicionalmente se anali- ocurrencia de GC a través de un modelo probit y los principales rubros de GB de los HGCS.

\section{Método}

El GC en salud se estimó según los datos de la Encuesta Nacional de Ingreso y Gasto de los Hogares (ENIGH) 2002-20148. Para determinar el porcentaje de HGCS se utiliza la metodología definida por la Unidad de Análisis Económico de la Secretaría de Salud (SS) basada en criterios definidos por la OMS en 2005, consensuada por la Comisión Nacional de Protección Social en Salud, FUNSALUD y la Dirección General de Evaluación del Desempeño de la $S^{9,10}$. Además de la ENIGH, entre los insumos para la estimación del indicador se encuentran la definición de ingresos y gastos corrientes totales del Instituto Nacional de Geografía y Estadística, y la línea de pobreza alimentaria definida por el Consejo Nacional de Evaluación de la Política Social11 en los años respectivos. La fórmula de cálculo de los HGCS es la siguiente:

$$
h g c_{i}=\left\{\begin{array}{l}
0, c f h_{i}<30 \% \\
1, c f h_{i} \geq 30 \%
\end{array}\right.
$$

donde $h g c_{i}$ son los hogares con GC ycfh es la capacidad financiera de los hogares. La contribución financiera del hogar al gasto en cuidados de la salud es un indicador de la carga financiera generada por gastos en salud, que es igual al gasto en salud del hogar (gsalh) entre la capacidad de pago del hogar definida como los ingresos monetarios efectivos del hogar $\left(c p h_{i}\right)$, que están por encima del nivel de subsistencia.

El análisis se realizó para el periodo 2002-2014. Adicionalmente, se estimó un modelo probit para identificar los factores asociados a una mayor probabilidad de ocurrencia de GC. La especificación del modelo se presenta en la siguiente ecuación:

$$
E\left(Y_{i}=\operatorname{Prob}\left(Y_{i}=1\right)=M_{i}=\frac{e^{\alpha+\beta_{k} X_{k i}}}{1+e^{\alpha+\beta_{k} X_{k i}}}\right.
$$

donde:

Mi=probabilidad del estado

$$
\begin{gathered}
M_{i}+M_{i} e^{\alpha+\beta_{k} X_{k i}}=e^{\alpha+\beta_{k} X_{k i}} \\
M_{i}=\left(1-M_{i}\right) e^{\alpha+\beta_{k} X_{k i}} \\
\frac{M_{i}}{\left(1-M_{i}\right)}=e^{\alpha+\beta_{k} X_{k i}}, \text { odds ratio }
\end{gathered}
$$

Se integraron en el análisis aspectos concernientes a la cobertura efectiva relacionada con la utilización de servicios públicos y la evolución del ingreso de los hogares en México. 


\section{Resultados}

\section{Sociodemográficos}

La Tabla 1 muestra algunas características sociodemográficas y económicas de la población mexicana en el periodo 2002-2014. Se observó un incremento de la población de poco más de 18.9 millones de personas en todo el periodo. La estructura por sexo se ha mantenido relativamente constante en estos años, con un $49 \%$ de hombres y un $51 \%$ de mujeres. Respecto al tamaño de la localidad, el $78 \%$ de los hogares se encontraban concentrados en áreas urbanas y el $22 \%$ en zonas rurales. De acuerdo con el Instituto Nacional de Información Estadística y Geográfica (INEGI), las localidades urbanas son aquellas que concentran 2500 o más habitantes, mientras que las localidades rurales son aquellas con menos de 2500 habitantes; esta composición se ha mantenido estable a lo largo del periodo.

Se observó un aumento importante en el número de hogares, al pasar de 24.6 millones en 2002 a 31.7 millones en 2014, lo que representó un aumento del $28.5 \%$ (siete millones de hogares). Por otra parte, la estructura interna del hogar se mantuvo relativamente constante: un integrante del hogar tiene menos de 14 años, 2.4 integrantes tienen entre 14 y 64 años, y 0.3 integrantes tienen 65 años o más. El tamaño promedio del hogar disminuyó en el periodo de $4.1 \mathrm{a}$ 3.8 integrantes. En 2014, en promedio, el $52.6 \%$ de los hogares reportaron algún gasto en salud.

Respecto a la afiliación reportada, se asignó al hogar la afiliación del jefe del hogar; dicho análisis se pudo realizar a partir de 2008. Esta restricción se vincula con la forma en que se pregunta en la encuesta $y$, en parte, con el hecho de que el SPSS aún no alcanzaba los niveles de cobertura previstos en todas las entidades federativas. Entre 2008 y 2014, los hogares que contaban con aseguramiento en salud por el Instituto Mexicano del Seguro Social (IMSS) se mantuvieron relativamente constantes: un $32 \%$ en el periodo. Por otra parte, los hogares cubiertos por el SPSS pasaron del 17\% en 2008 al 39\% en 2014. Finalmente, los hogares que no están cubiertos por ninguna institución pública de salud se redujeron conforme se incrementó la cobertura del SPSS, al pasar del $37 \%$ en 2008 al $18 \%$ en 2014.

Cabe señalar la evolución del ingreso y del gasto neto total promedio de los hogares para el periodo de análisis, ya que presentaron una tendencia a la baja a partir de 2008, en gran medida influenciada por el ciclo económico a partir de la crisis mundial de 2008.

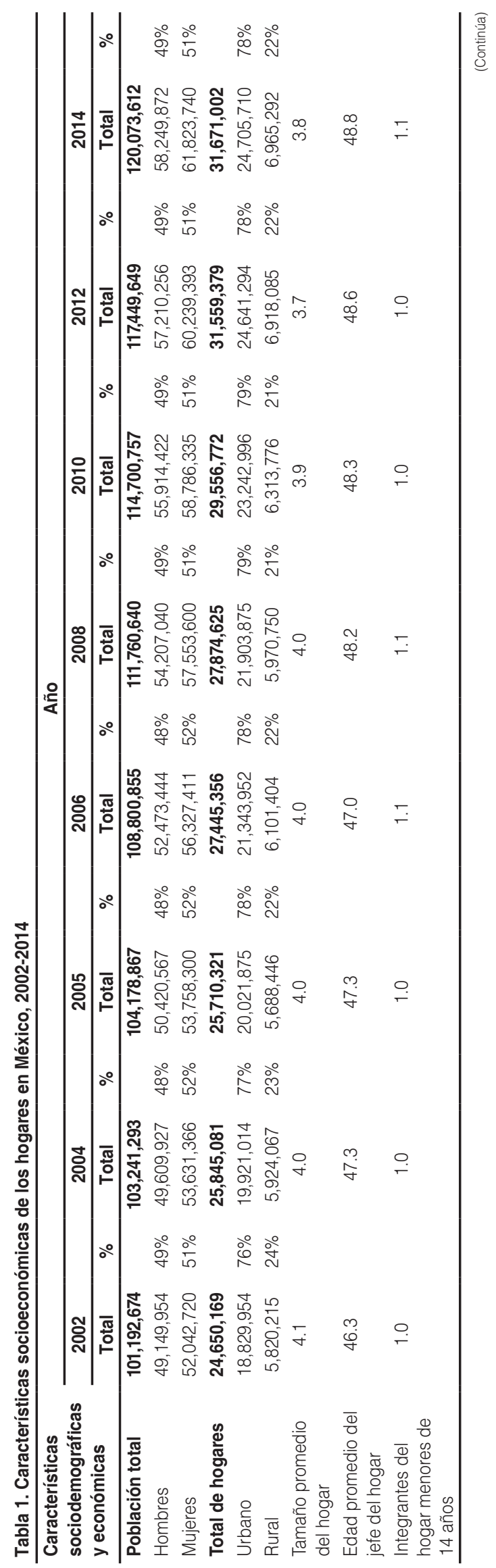




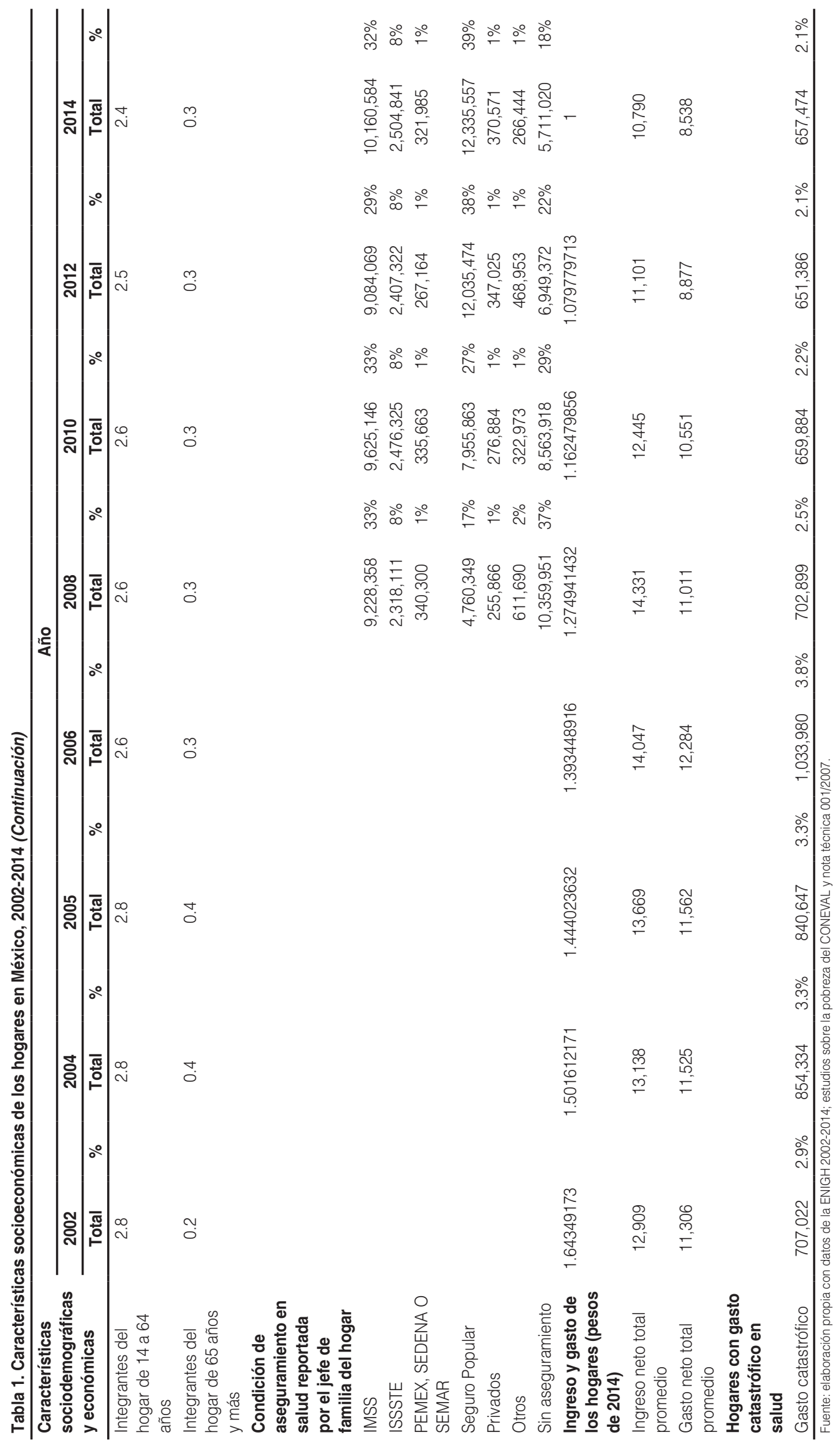


Es necesario explorar con mayor profundidad el efecto del ciclo económico en la disminución o el aumento del gasto en salud, y en consecuencia del GC, para poder evaluar el efecto real de la disminución del GC atribuible a las reformas en salud implementadas en los últimos años en México.

\section{Gasto catastrófico por motivos de salud}

En la figura 1 se presentan los resultados de la estimación del porcentaje de HGCS para el periodo 2002-2014. Se observó una tendencia al alza en el periodo 2002-2006, que se revierte para los años 2008-2014. En 2012, el porcentaje de HGCS se estimó en un $2.06 \%$, mientras que para 2014 se estimó en un $2.08 \%$. Esto implica que hubo un incremento de poco más de 6000 hogares (equivalente al 1.0\%) que presentaron GC en salud entre 2012 y 2014.

La reducción del porcentaje de HGCS a partir de 2006 es modesta, y debe considerarse que dicha reducción se presenta en un entorno socioeconómico adverso. Lo anterior se ve reflejado en un incremento del $15.4 \%$ en el número de hogares de 2006 a 2014 , así como una reducción en el ingreso neto total promedio de los hogares. Esta tendencia puede estar vinculada, entre otras razones, con la creación y el incremento en la cobertura de la afiliación de la población a través del SPSS. La tendencia a la baja del GC entre 2008 y 2014 coincide con la tendencia decreciente del ingreso neto total de los hogares, lo que explica la reducción observada de HGCS (Fig. 2). Con la información que proporciona la ENIGH es difícil establecer si la disminución del GC se debe al aumento de la cobertura pública a través del SPSS o a la disminución del ingreso de los hogares.

El porcentaje de población con aseguramiento público que utiliza servicios públicos de atención médica es un indicador de seguimiento del Programa Sectorial de Salud (PROSESA) 2013-2018 ${ }^{12}$. Al relacionar dicho indicador con el porcentaje de HGCS entre aquellos hogares que cuentan con aseguramiento público, puede valorarse indirectamente la disminución del GC atribuible al aumento de la protección financiera pública en salud. Como se observa en la Fig. 2, existe una relación inversa entre el indicador de cobertura efectiva y el indicador de GC.

Se encontró una relación directa entre el ingreso neto total de los hogares y el monto designado a gasto en salud, y una relación inversa entre la cobertura efectiva del aseguramiento público y el porcentaje de HGCS. La disminución de este último puede atribuirse

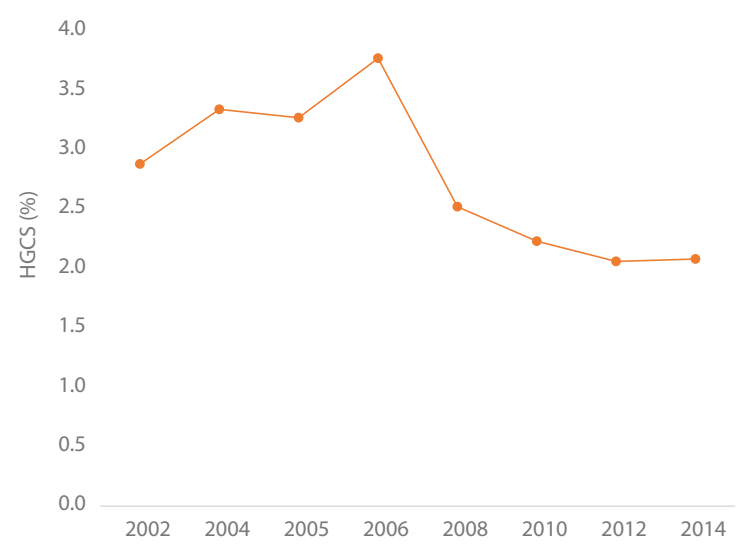

Figura 1. Hogares con gasto catastrófico (HGCS) por motivos de salud, 2002-2014. Fuente: elaboración propia con datos de la ENIGH 2002-2014; estudios sobre la pobreza del CONEVAL y nota técnica $001 / 2007$.

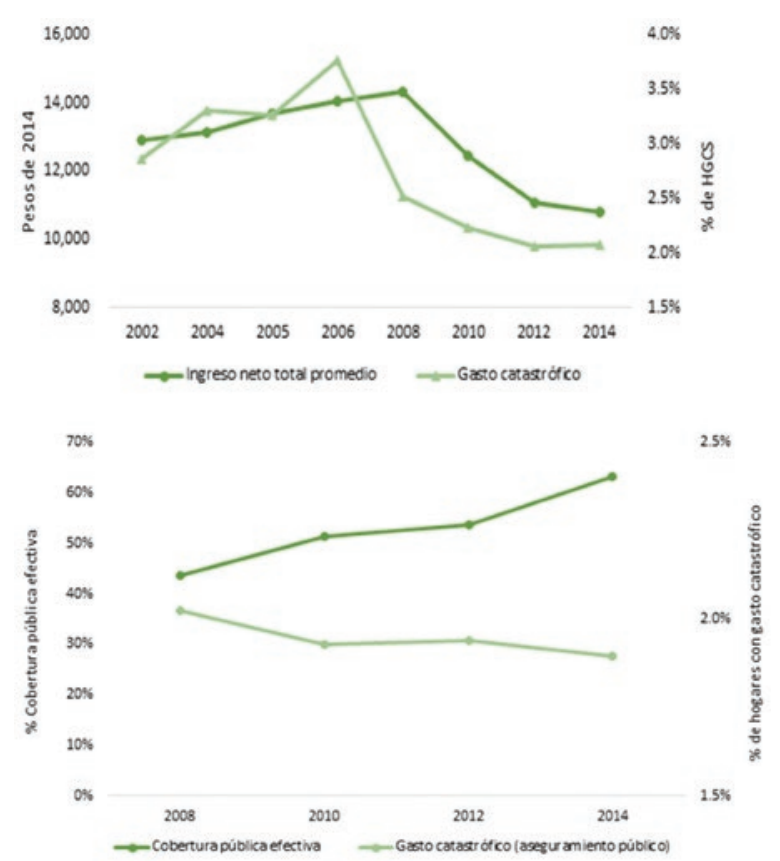

Figura 2. Ingreso neto total, cobertura pública efectiva y gasto catastrófico en salud, 2002-2014. Fuente: elaboración propia con datos de la ENIGH 2002-2014; estudios sobre la pobreza del CONEVAL y nota técnica 001/2007.

a un efecto combinado de la baja en el ingreso y el aumento de la cobertura pública. El aumento en la cobertura pública a través del SPSS redujo el impacto de la reducción del ingreso en el GC en salud.

\section{Gasto catastrófico según el nivel de ingreso de los hogares, la afiliación reportada y la condición rural o urbana}

La desagregación del GC considerando el nivel de ingreso de los hogares y la condición rural o urbana 
mostró en 2014 que los hogares que se ubican en los menores deciles de ingreso presentaron mayor incidencia de GC (quintil I: 4.53\%; quintil II: $2.0 \%$ ). De igual forma, en 2014, los hogares rurales tuvieron mayor proporción de HGCS (3.1\%) que los urbanos $(1.8 \%)$.

Tomando en consideración que el cálculo del GC se realiza por hogar, para poder analizar la relación entre la ocurrencia de GC, el nivel de ingreso y la condición de aseguramiento se identificó la afiliación de cada hogar mediante la afiliación reportada por el jefe del hogar en la ENIGH. Al desagregar el indicador de HGCS según la afiliación reportada por el jefe del hogar se observó que en los hogares con aseguramiento del SPSS se redujo el porcentaje de HGCS del $3.47 \%$ en 2008 al $2.49 \%$ en 2014 . En el IMSS, la disminución fue del 1.41 al 1.17\%. En el ISSTE hubo un incremento del 1.53 al $1.93 \%$, y para la población sin afiliación hubo una disminución del 3.39 al 2.76\%.

Al analizar el indicador según el quintil de ingreso y la condición de aseguramiento, destacan los incrementos observados para los hogares del primer quintil con aseguramiento en el Instituto de Seguridad y Servicios Sociales de los Trabajadores del Estado (ISSSTE), ya que de 2008 a 2014 pasó del 3.4 al $12.0 \%$, lo que implicó un aumento de 10,000 hogares con dicha condición. En el IMSS, para el primer quintil se observó un incremento del $2.33 \%$ en 2008 al $3.30 \%$ en 2014. Para los hogares con afiliación del SPSS en el primer quintil se observó una disminución del $4.95 \%$ en 2008 al $3.82 \%$ en 2014 . Por último, los hogares sin afiliación reportada presentaron un incremento en el GC del primer quintil de ingreso del 5.46 al $6.73 \%$.

\section{Gasto catastrófico por entidad federativa}

Aun cuando la ENIGH no es representativa por entidad federativa, se presenta de manera informativa el porcentaje de HGCS comparando los años 2006 y 2014, antes y después de la implementación nacional del SPSS (Fig. 3). En 2006, ocho entidades presentaron un porcentaje de HGCS en un rango del 4 al $7 \%, 15$ en un rango del 2 al $4 \%$, y nueve entre el 1 y el $2 \%$. Para 2014, el panorama cambió significativamente: 24 entidades se ubicaron en un rango del 1 al $2 \%$, seis entre el 2 y el $3 \%$, una entre el 3 y el $4 \%$, y una entre el 4 y el $6 \%$ (Fig. 3). Las entidades federativas con mayor porcentaje de HGCS en 2014 fueron Guanajuato (3.9\%), Oaxaca (3.5\%) y Michoacán $(5.8 \%)$, y las entidades con menor porcentaje fueron
Nuevo León $(0.71 \%)$, Sonora $(0.73 \%)$ y Estado de México (0.75\%). De 2006 a 2014, 27 entidades federativas presentaron una disminución promedio del $48 \%$ en el indicador y cinco tuvieron un incremento promedio del $46 \%$.

\section{Factores asociados a la ocurrencia de gasto catastrófico}

Se estimó un modelo probit para determinar los factores que inciden en la probabilidad de que un hogar incurra en GC. El modelo probabilístico con variable dependiente binaria consideró todas las características del hogar disponibles en la ENIGH. Sin embargo, mediante el proceso de validación el modelo final contempló como variables significativas la afiliación del hogar y si el hogar cuenta con miembros con enfermedades crónicas (este indicador se identificó a través del gasto en medicamentos relacionados con diabetes e hipertensión); también se consideraron el gasto en hospitalización y la condición rural o urbana del hogar. Por último, se contempló el nivel de ingreso mediante la estimación de las líneas de pobreza definidas por SEDESOL: alimentaria, de capacidades y patrimonial (Tabla 2).

El modelo probit estimado fue significativo y eficiente al clasificar los HGCS a partir del modelo con $\operatorname{Pr}(\mathrm{D}) \geq 0.5)$; el $98.19 \%$ de los casos fueron clasificados correctamente. Los factores determinantes para que un hogar incurra en GC fueron la afiliación, los hogares con miembros con enfermedades crónicas, los hogares con gasto en hospitalización, la condición rural y el hecho de que el hogar se encuentre por debajo de la línea de pobreza alimentaria. Los signos de los coeficientes muestran que la afiliación es un factor protector para desincentivar el GC; contar con alguna afiliación disminuye la probabilidad de incurrir en GC. Por otro lado, los hogares con miembros que padecen enfermedades crónicas tienen mayor probabilidad de incurrir en GC, así como aquellos que presentaron gasto hospitalario. La condición rural influyó positivamente en la probabilidad de ocurrencia de GC. Por último, en cuanto a las líneas de pobreza, la pobreza alimentaria fue significativa, por lo que evidentemente es un determinante en la ocurrencia de GC.

Los efectos marginales mostraron que la afiliación a alguna institución de salud disminuye la probabilidad de incurrir en GC un $0.5 \%$, la presencia de individuos con enfermedades crónicas aumenta un $8 \%$ la probabilidad de GC, la condición rural la aumenta un 


\section{6}

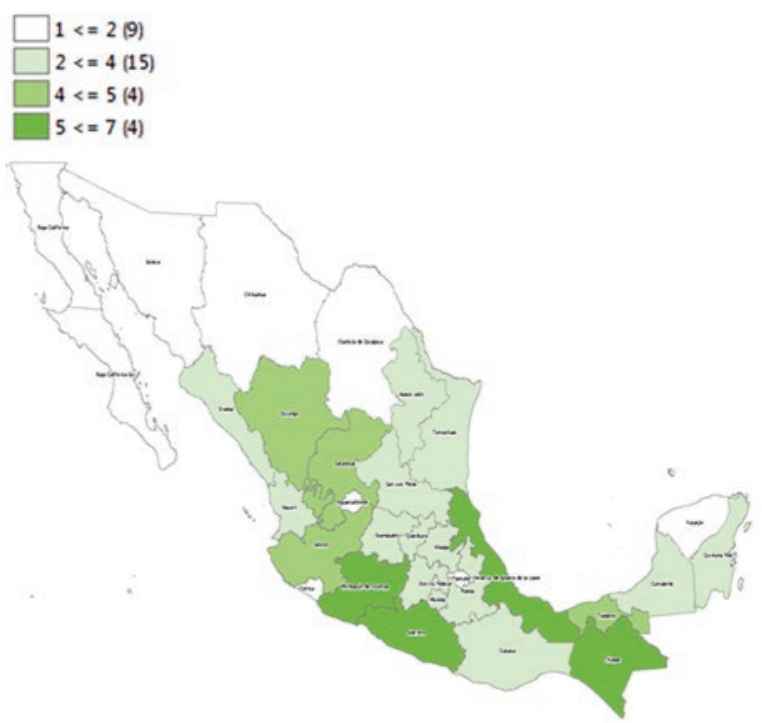

\section{4}

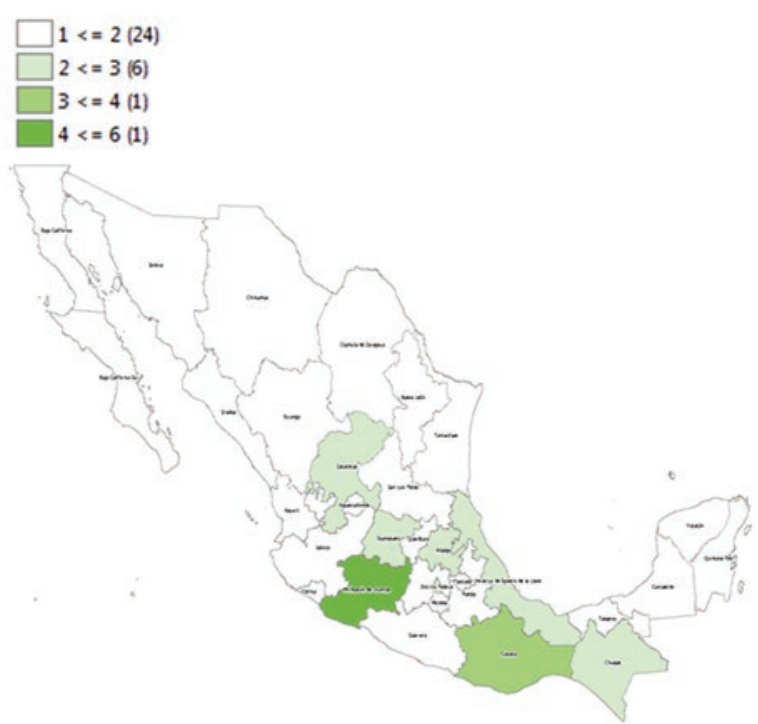

Figura 3. Hogares con gasto catastrófico (GC) por entidad federativa. El mapa presenta el rango del porcentaje de GC en que se ubican las entidades federativas $(x \leq y)$, y entre paréntesis se indica el número de entidades federativas que se encuentran en dicho rango. Fuente: elaboración propia con datos de la ENIGH 2002-2014, estudios sobre la pobreza del CONEVAL y nota técnica 001/2007.

Tabla 2. Modelo probit de gasto catastrófico: efectos marginales

\begin{tabular}{|c|c|c|c|c|c|c|}
\hline Variable & $\mathrm{dy} / \mathrm{dx}$ & EE & $\mathbf{z}$ & $P>z$ & \multicolumn{2}{|c|}{ IC $95 \%$} \\
\hline Afiliación* & -0.0058381 & 0.00254 & -2.29 & 0.022 & -0.010826 & -0.000851 \\
\hline Crónicas* & 0.0800964 & 0.01349 & 5.94 & 0.000 & 0.053649 & 0.106544 \\
\hline Hospitalización* & 0.0000274 & 0.00000 & 8.92 & 0.000 & 0.000021 & 0.000033 \\
\hline Condición rural/urbana* & 0.0090252 & 0.00224 & 4.04 & 0.000 & 0.004643 & 0.013407 \\
\hline Línea de pobreza alimentaria* & 0.0076359 & 0.00398 & 1.92 & 0.045 & -0.000156 & 0.015428 \\
\hline
\end{tabular}

*Significativo al 95\% de confianza.

Número de observaciones: 19,479; Wald $\chi^{2}$ (5) 268.60; Prob > $\chi^{2}$ (0.0000); Log likelihood: 1367.0439; probabilidad de los efectos marginales: $98.19 \%$.

Fuente: elaboración propia con datos de la ENIGH 2002-2014, estudios sobre la pobreza del CONEVAL y nota técnica 001/2007.

$0.9 \%$, estar por debajo de la línea de pobreza alimentaria un $0.7 \%$, y gastar en hospitalización contribuye de manera positiva con un $0.002 \%$. Si bien el gasto hospitalario tiene un efecto en la probabilidad de ocurrencia de GC, este efecto es marginal (el gasto en hospitalización no necesariamente implica GC, lo que puede explicarse porque en general el gasto en hospitalización fue en atención del parto).

La desagregación del modelo por institución de afiliación muestra que aquellos hogares que reportaron estar afiliados al IMSS o a seguros privados, y aquellos que no cuentan con afiliación alguna, fueron los hogares con mayor probabilidad de incurrir en GC. En el caso de los hogares con pobreza de capacidades y patrimonial, los resultados no fueron estadísticamente significativos. Por otra parte, el análisis del GB por rubro en 2014 indica que la erogación en medicamentos representa la mayor proporción (61.3\%: $33.4 \%$ medicamentos recetados y $27.9 \%$ medicamentos sin receta), seguida de consulta externa $(25.2 \%)$, otros $(7.9 \%)$, embarazo y parto $(4.1 \%)$, y hospitalización $(2.1 \%)$. Esta tendencia en la concentración del gasto en medicamentos se mantiene al analizar la condición de aseguramiento público en salud y el tipo de gasto realizado. Sin embargo, cabe mencionar que, para el SPSS, el IMSS y el ISSSTE, la proporción de gasto en medicamentos recetados en 2014 disminuyó respecto a 2006.

\section{Discusión}

Los resultados del análisis del porcentaje de HGCS en México indican que existe una relación con el ingreso de los hogares, la cobertura efectiva de 
instituciones públicas, la condición de aseguramiento, el tamaño de la localidad y el rubro de gasto en salud. Destaca el hecho de que, aun con la expansión de la cobertura pública de salud en 2014 , el $52.6 \%$ de los hogares reportaron GB en salud y el $2.08 \%$ GC.

A partir de los resultados es posible identificar que vivir en una zona rural influye significativamente en que los hogares incurran en GC. El análisis por condición de aseguramiento sugiere que el déficit en la protección financiera continúa presente, independientemente de la condición de aseguramiento público. Sin embargo, es necesario evaluar el efecto de sustitución en la demanda de salud, pues es probable que, al aumentar la cobertura pública, los hogares destinen un porcentaje de su ingreso a rubros de salud no cubiertos por los esquemas de aseguramiento público. Cabe destacar que un hogar cubierto por el SPSS en el primer y último quintil de ingreso tiene una mayor probabilidad de incurrir en GC en salud. Sin embargo, es necesario reconocer que los hogares cubiertos por el SPSS presentaron entre 2008 y 2014 una reducción significativa en GC. Para el primer quintil de ingreso, los HGCS muestran una tendencia a la baja, congruente con los objetivos establecidos en el PROSESA 2013-2018. Estos resultados dan pauta para considerar que la expansión del aseguramiento público en salud, si bien coadyuva en la reducción del número de hogares en situaciones extremas en gastos de salud, no es suficiente para incidir en un decremento absoluto en el GC en salud. Por lo anterior, es necesario evaluar el efecto de la baja en el ingreso y el aumento de la cobertura efectiva del aseguramiento público en salud como posibles causas de la reducción del porcentaje de HGCS en México.

En la mayoría de las entidades federativas hubo una reducción del porcentaje de HGCS entre 2006 y 2014. Si bien ha habido avances importantes, aún se mantiene el reto de reducir el porcentaje de HGCS en aquellas entidades que presentaron porcentajes del 3 hasta el $6 \%$. Adicionalmente, la presencia de enfermedades crónicas representa una carga financiera importante que incrementa la probabilidad de incurrir en GC.

El papel del GC en el Sistema Nacional de Salud mexicano indica la persistencia de inequidades tanto en el financiamiento como en el acceso a servicios de salud, particularmente a medicamentos. Estas inequidades deben ser analizadas en un contexto intersectorial.

\section{Bibliografía}

1. Comisión Mexicana sobre Macroeconomía y Salud. Macroeconomía y salud: invertir en salud para el desarrollo económico. México: Fondo de Cultura Económica, Secretaría de Salud, Fundación Mexicana para la Salud, Instituto Nacional de Salud Pública, Secretaría de Hacienda y Crédito Público, Comisión Mexicana sobre Macroeconomía y Salud; 2006.

2. OECD (2013), Health at a Glance 2013: OECD Indicators, OECD Publishing. Disponible en: http://dx.doi.org/10.1787/health_glance-2013-en

3. Kelly MP, Morgan A, Bonnefoy J, et al. The social determinants of health: developing an evidence base for political action. Universidad del Desarrollo, Chile, and National Institute for Health and Clinical Excellence, United Kingdom; 2007 (Final Report to Commission on the Social Determinants of Health of the World Health Organization).

4. Organización Mundial de la Salud. Subsanar las desigualdades en una generación: alcanzar la equidad sanitaria actuando sobre los determinantes sociales de la salud. Informe final de la Comisión sobre los Determinantes Sociales de la Salud. Geneva: OMS; 2009.

5. Knaul F, Arreola H, Méndez O. Protección financiera y gasto catastrófico de los hogares en Caleidoscopio de la Salud. México D.F.: Funsalud; 2003.

6. Knaul FM, Arreola H, Méndez O, et al. Las evidencias benefician al sistema de salud: reforma para remediar el gasto catastrófico y empobrecedor en salud en México. Salud Publica México. 2007; 49(Supl 1):S70-S87.

7. González E, Barraza M, Gutiérrez C, et al. Sistema de Protección Social en Salud: elementos conceptuales, financieros y operativos. México: Fondo de Cultura Económica, Secretaría de Salud, Fundación Mexicana para la Salud, Instituto Nacional de Salud Pública; 2006.

8. Instituto Nacional de Estadística y Geografía - INEGI. Encuesta Nacional de Ingreso y Gasto de los Hogares 2002-2014. ENIGH, 2002-2014. México, 2015.

9. Secretaría de Salud. Rendición de Cuentas en Salud 2008-2012. Dirección General de Evaluación del Desempeño, Subsecretaría de Integración y Desarrollo del Sector Salud. México, 2013.

10. Unidad de Análisis Económico. Metodología para la estimación del gasto catastrófico y empobrecedor en salud de los hogares mexicanos. México: Secretaría de Salud; 2009.

11. CONEVAL. Medición de la pobreza en México: metodología para la medición de la pobreza por ingresos. 2002-2014 CONEVAL. México; 2015.

12. Secretaría de Salud. Programa Sectorial de Salud 2013-2018. México; 2013. 